Vol. 8, Issue 5, May 2019

\title{
Stationed Automotive's Surveillance of Streets for Smart City
}

\author{
Amogh H Kashyap ${ }^{1}$, Chethan A ${ }^{2}$, Anusha C Shetty ${ }^{3}$, Dhatri R Bidarahalli ${ }^{4}$ \\ R\&D Engineer, Nokia Solutions and Networks India Pvt Ltd, Bengaluru, India ${ }^{1,2,3,4}$
}

\begin{abstract}
Traffic congestion is a common hindrance which is caused mainly due to automobiles parked in "No Parking" areas knowingly or unknowingly. Analogously, the police vehicles need to monitor these areas which further adds pollution and congestion into the system. Therefore, we propose a Deep Learning based solution which can monitor vehicular movements. This involves vehicle and number plate recognition through which the owner's details are fetched, and warning messages are sent after a certain time specified by the governing body. Meanwhile, the local police authorities are notified about the wrongly parked vehicle, allowing them to easily tow it, thus saving a lot of human and fuel resources which also in the process reduces the pollution. A Graphical User Interface is provided at the local police stations to display various traffic violations and status across the city.
\end{abstract}

Keywords: Deep Learning, Traffic Monitoring System

\section{INTRODUCTION}

One of the main reasons for traffic congestion is due to automobiles being parked in no parking zones across the city. The local police authorities go on patrols to inspect and tow the vehicles parked in no parking areas. This is a tedious process for them and results in the wastage of fuel resources. Since many areas of the city already have CCTV cameras installed, these cameras can be used to extract information about the parked vehicles. These cameras can be used to locate the vehicles in the no parking zones. Using Deep learning-based Object detection models, the automobiles can be detected, and the number plate can be read using an ALPR system. The number plate details can then be used to fetch the details of the owner from the RTO Database. The owner can now be warned by sending messages to his/her contact number via any messaging platform tied to a carrier service provider. On the other hand, the local police station can be equipped with a GUI display with the details of vehicles identified in no parking area. The system first warns the user to move the vehicle and if the vehicle is still stationed at that spot, a penalty is levied, and a ticket is registered at the nearest police station identified using the location of the CCTV camera and the owner is intimated about the same. If the vehicle is not moved even after the second warning, towing procedure is initiated at the local police station near to that location. Thus, this system reduces the burden on traffic authorities and helps in reducing the traffic congestion along with pollutions caused by the automobiles.

\section{PROPOSED SOLUTION}

The solution we have come up is a Deep learning-based approach using computer vision techniques such as object detection and tracking.

Detection- Every area will have few No-parking area zones and the multitude of CCTV Cameras in such locations can be utilized. The cameras already present on the roads are used to fetch the live stream which is available in RTSP and RTMP streams. A device management platform (IMPACT) is used to take inputs from multiple such cameras by uniquely identifying devices and hold necessary telemetry information. The "YOLO v2 Real-Time Object Detection" [1] neural network is a neural networked based algorithm, which is used for vehicle detection. This algorithm takes in the video feed, which is then split into frames and fed to the networks. Then each frame is divided into regions and prediction is done on each of these regions to get bounding box and region probabilities. The interframe tracking is done using Kalman filters in order to avoid redundancies in detections. After the vehicles of interest are identified, the number plate is read using "OpenALPR" network [2][3]. This live information is continuously sent to the central monitoring system and updated on the graphical user interface. REST/HTTP interface is used to upload the data to the monitoring server on the cloud platform.

Monitor- The Centralized monitoring system takes the live feed and detection details from detection module and is responsible for all other actions in the system. The number plate details obtained from detection model is then sent to an API exposed by the Regional Transport Office (RTO), which then fetches the details (such as contact number and registered address) of the detected vehicles' owner. These RTO API's exposed are accessed through gRPC interface [4]. 
Vol. 8, Issue 5, May 2019

This is a more reliable, secure and faster communication protocol as it involves header compression and HTTP2.0. Thus, from this the owner's details such as contact number or/and address is fetched.

SMS- The duration for which a vehicle can be parked in no-parking area can be specified by the governing body. The warning messages are sent to the registered contact number using NEX[6] (Network Exposure Functions), which can help inform the owner of the vehicle to remove his car. Alternatively, various carrier service providers can be used to send messages. If the vehicle is not removed even after a specified duration, penalty as set by traffic authority is levied and a message related to this is also sent to the owner of the car and the status is updated to the Graphical user interface by the controller platform. The NEX module is kept independent from the controller platform to allow easier integration with other service providers. The notifications to send SMS are received over REST interface with authenticated tokens.

UI- Monitoring system transmits the information about the automobiles to the GUI and updates the live status continuously over websockets[7]. The police stations in the vicinity have access to a UI, which displays the map of the area and vehicles of interest. The vehicles status can be differentiated by different colours, i.e. the time for which they are in no-parking areas. The vehicles are displayed as pins on Google maps which, on click, will show the status of the vehicle.

State I: Vehicle detected in No-parking area and available nearby parking area co-ordinates are sent to car owner. State II: Vehicle not moved after the wait time, penalty levied, and ticket details are sent to car owner.

State III: Vehicle not moved after the wait time, penalty. Towing request is sent to nearest station.

Traffic authority can now see and get to know the exact location of the vehicles which are to be towed away

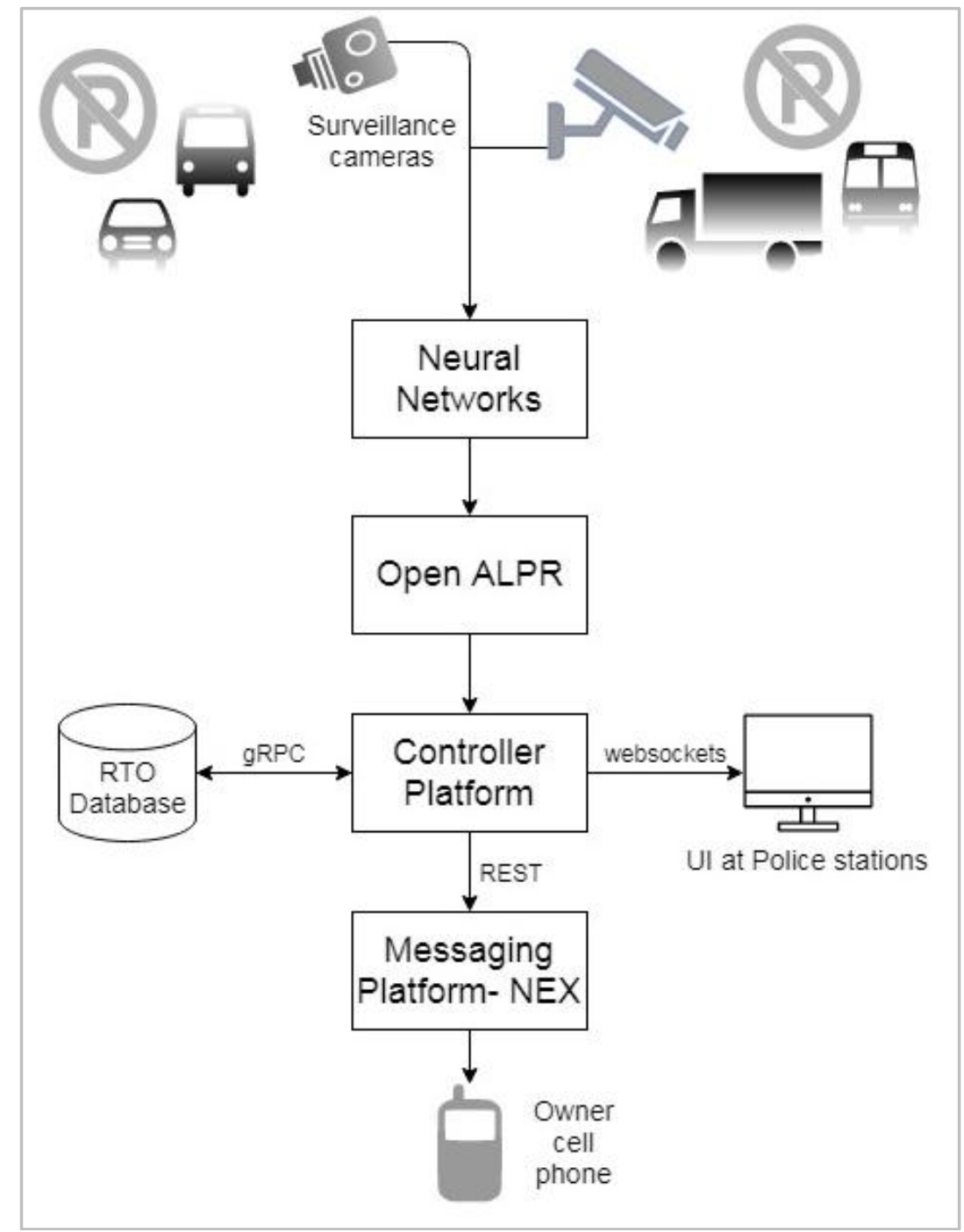

Fig. 1 Block diagram of the Solution system 

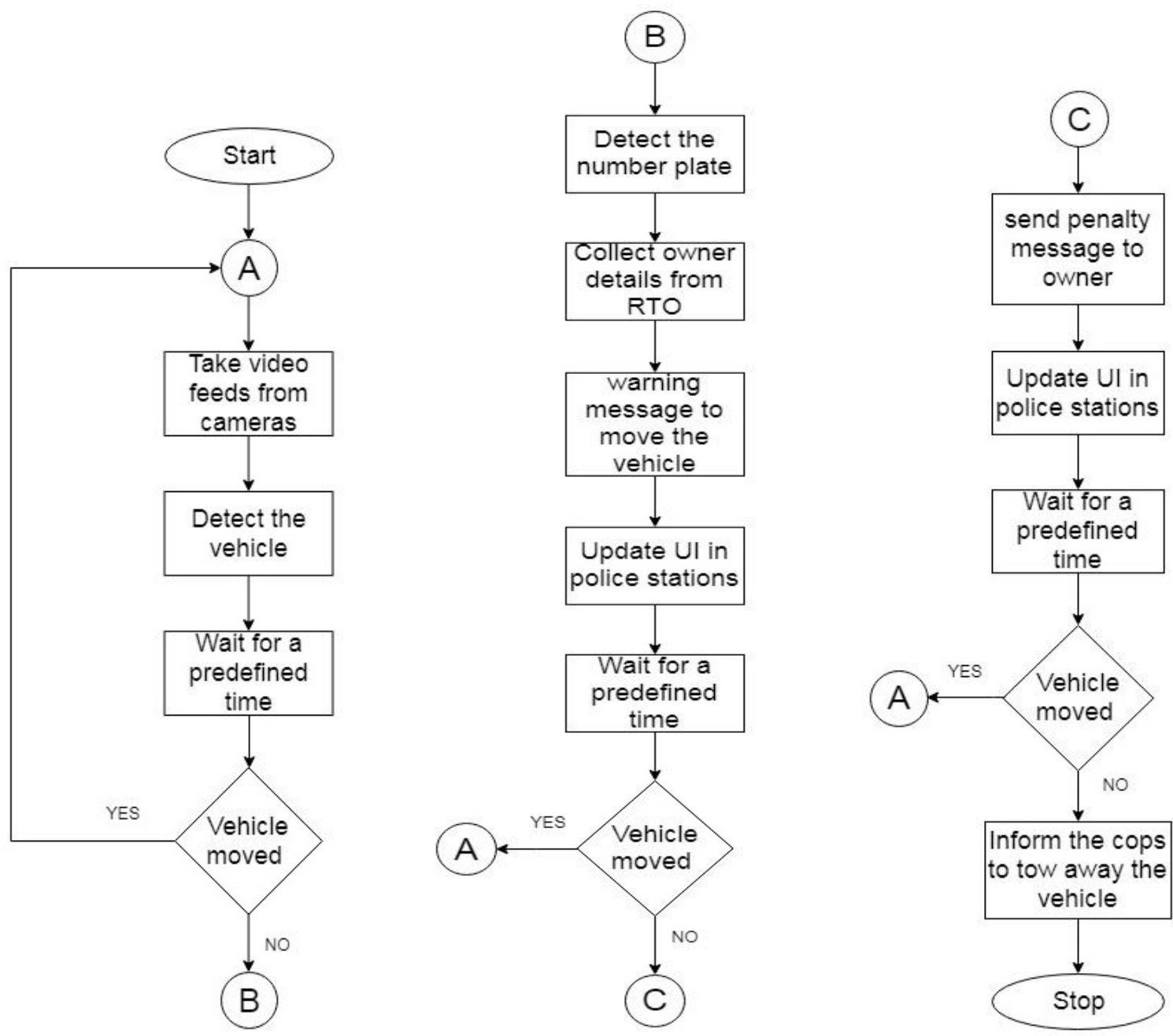

Fig. 2 Flow chart of the Solution system

\section{RESULTS AND CONCLUSION}

This is an effective solution which can help reduce traffic as well as save fuel and Human resources, which are generally wasted in patrolling activities. The Deep learning-based approach makes this solution more reliable and the usage of the existing cameras makes it a cost-effective solution. This solution eliminates the need for the police to navigate the neighbourhood for improperly parked vehicles, thus saving time and effort. Therefore, by towing away wrongly parked vehicles along with automation of the ticketing system, the traffic congestion can be reduced considerably.

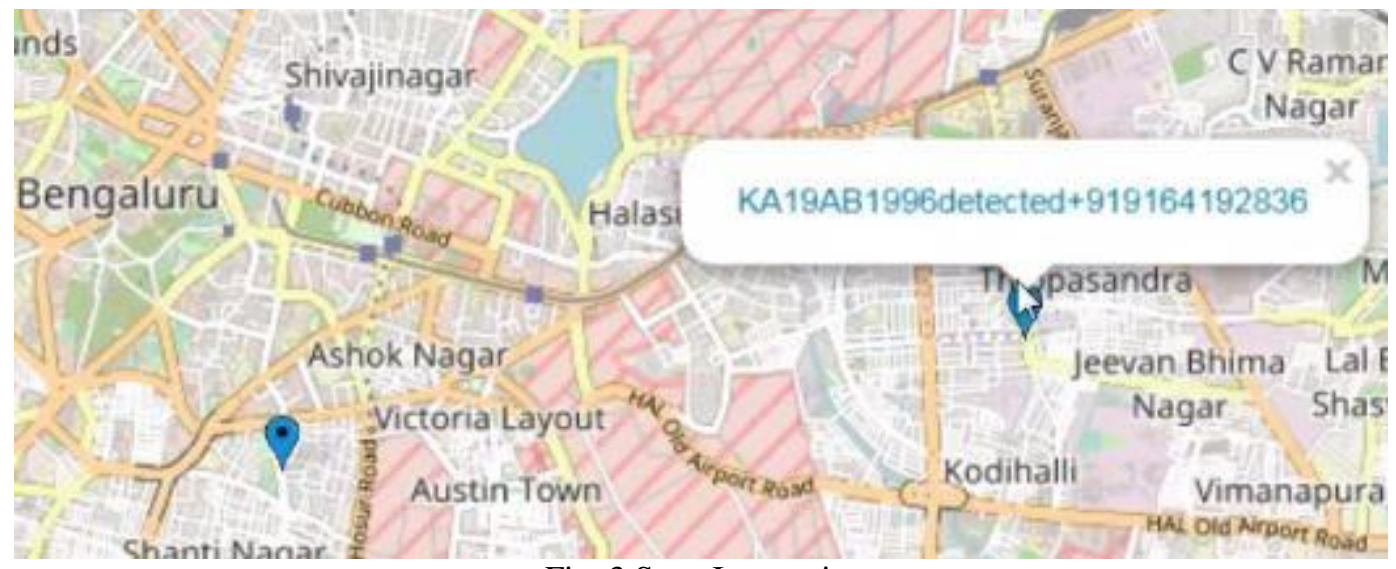

Fig. 3 State I scenario 
Vol. 8, Issue 5, May 2019

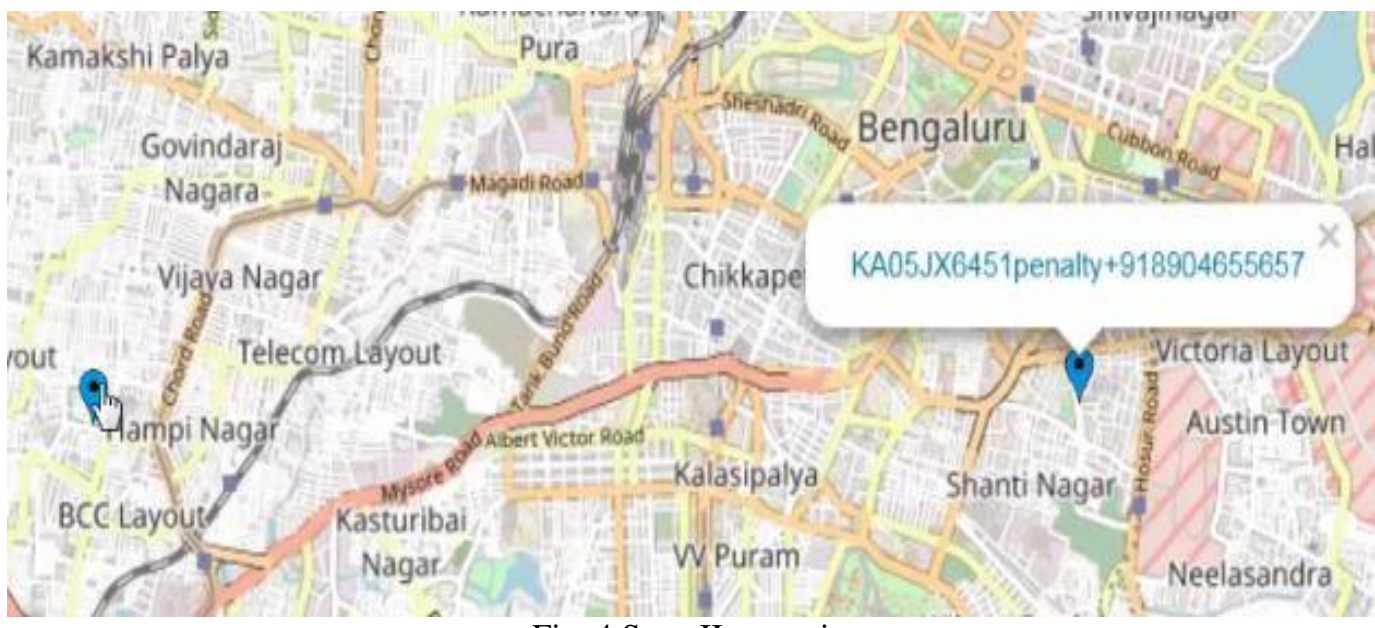

Fig. 4 State II scenario

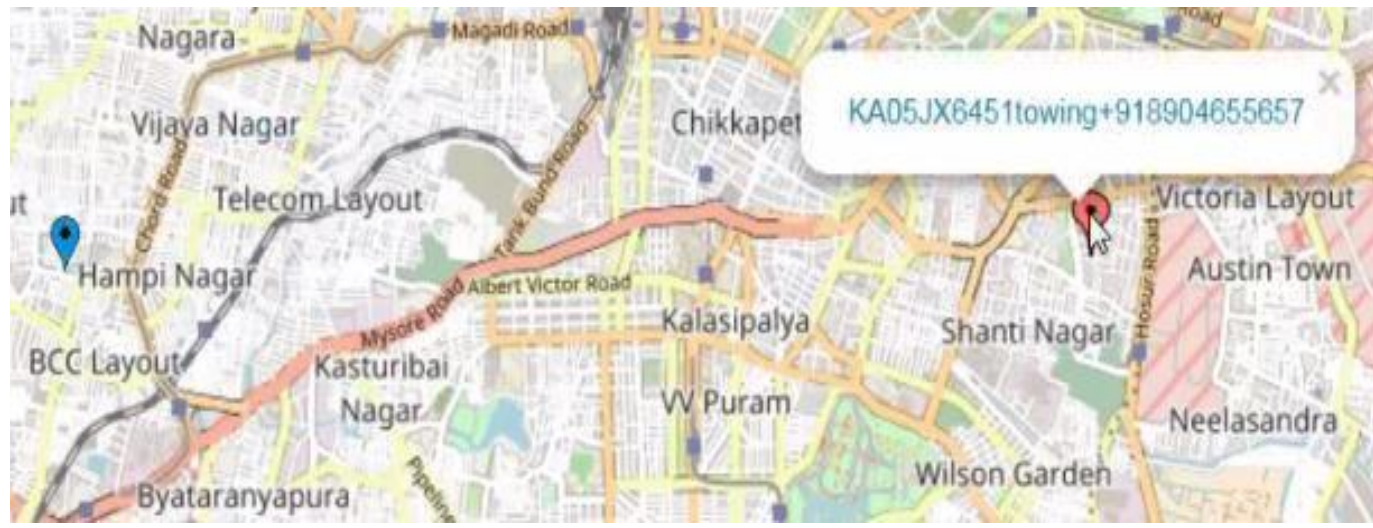

Fig. 5 State III scenario

\section{FUTURE WORK}

We would like to extend this technology to help locate vehicles which break down on the highways. The details of such vehicles can be intimated to towing services who can take immediate actions and avoid traffic, thus aiding the car owners in locating towing services or mechanics to expedite a quick resolution to the breakdown. This system can also be deployed on drones installed with a camera which could be flown across the city to find the vehicles of interest.

\section{REFERENCES}

[1]. Rachel Huang, Jonathan Pedoeem, Cuixian Chen, "YOLO-LITE: A Real-Time Object Detection Algorithm Optimized for Non-GPU Computers"arXiv:1811.05588v1 [cs.CV] 14 Nov 2018

[2]. https://github.com/openalpr/openalpr/wiki/OpenALPR-Design

[3]. Santosh G Kashid, Sanjay A Pardeshi, "Detection \& Identification of illegally parked vehicles at no parking area", IEEE explore, Nov. 2014

[4]. Wang, Xingwei \& Zhao, Hong \& Zhu, Jiakeng. (1993). GRPC: A Communication Cooperation Mechanism in Distributed Systems.. Operating Systems Review. 27. 75-86.

[5]. Andy Neumann ; Nuno Laranjeiro ; Jorge Bernardino, IEEE Transactions on Services Computing , 10.1109/TSC.2018.2847344, June 2018

[6]. Chao Geng ; Chun-Rong Sui ; Wanquan Dong," Research on web-Based SMS application", 10.1109/ICCIS.2012.266, Aug. 2012

[7]. D. G. Synodinos, "HTML 5 Web Sockets vs. Comet and Ajax," 2008. 